\title{
Reparo artroscópico das lesões completas isoladas do subescapular
}

\author{
Arthroscopic repair of complete, isolated \\ lesions of the subscapularis tendon
}

\author{
Niso Eduardo BalsinI ${ }^{1}$, Niso BalsinI ${ }^{1}$, LUCIANA Kochen ${ }^{1}$, LUCIANo José Gori PalKa ${ }^{1}$, \\ ANDRÉ VILELA' ${ }^{1}$, JASON SCHREINER DOS SANTOS ${ }^{2}$
}

\section{RESUMO}

Objetivo: Avaliar o resultado retrospectivo de 12 pacientes submetidos ao reparo artroscópico de lesões completas isoladas do tendão do subescapular com seguimento mínimo de um ano. Métodos: De 11 de abril de 2002 a 24 de setembro de 2004, realizou-se o reparo artroscópico de lesões completas do manguito rotador em 95 pacientes no Instituto Balsini. Pacientes com subescapular lesado somavam 31; 12 lesões isoladas compunham o grupo de estudo. Foram reavaliados com seguimento mínimo de um ano da cirurgia, considerando grau de elevação anterior ativa, escala da UCLA e satisfação do paciente. Resultados: A elevação anterior ativa préoperatória atingiu a média de $102^{\circ}$, o grau de elevação anterior ativa pós-operatória obteve a média de $175^{\circ}$. O ganho médio na elevação anterior ativa foi de $73^{\circ}$ (p < 0,0001). O UCLA pré-operatório teve a média de 15,25 pontos e o pós-operatório atingiu

* Trabalho realizado no Instituto de Ortopedia Niso Balsini - Joinville (SC), Brasil.

1. Médico Ortopedista do Instituto de Ortopedia Niso Balsini - Joinville (SC), Brasil.

2. Médico Ortopedista Estagiário do Instituto Balsini - Joinville (SC), Brasil.

Endereço para correspondência: Rua XV de Novembro, 1.156 - Centro - 89201-602 - Joinville (SC), Brasil. Tel./fax: (47) 3433-3835. E-mail: nisoedu@terra.com.br

Recebido: 30.5.06. Autor para revisão: 14.9.06. Reapresentação: 28. 8.08. Aprovado: 30.10 .08 .

Copyright RBO2008 a média de 31,66 pontos. $O$ índice da UCLA médio foi de 16 pontos ( $p<0,0001)$; 10 resultados excelentes, um bom e um mau. Resultados considerados satisfatórios foram 11 e um, insatisfatório. Conclusões: 1) A sutura artroscópica das lesões do subescapular apresenta resultados satisfatórios em $\mathbf{9 1 , 6 7 \% ~ d o s ~ c a s o s , ~ p a r a ~ a s ~ l e s o ̃ e s ~ i s o l a d a s ~ d o ~ t e r c ̧ o ~}$ superior e as lesões completas totais; 2) A instabilidade do bíceps é achado comum; 3) A sutura artroscópica do subescapular é possível mesmo nos tendões retraídos; 4) É uma técnica segura; 5) As lesões completas do terço superior do subescapular são sintomáticas e devem ser tratadas como tal.

Descritores - Ombro/lesões; Bainha rotadora/lesões; Artroscopia; Estudos retrospectivos

\section{ABSTRACT}

Objective: To evaluate the retrospective result of 12 patients submitted to arthroscopic repair of complete, isolated lesions of the subscapularis tendon with minimum follow-up of one year. Methods: From April 11, 2002 to September 24, 2004, the authors performed the arthroscopic repair of complete rotator cuff lesions in 95 patients at the Balsini Institute. Patients with lesioned subscapularis amounted to 31, and 12 isolated lesions were included in the study group. They were re-analyzed with a minimum followup of one year after surgery to evaluate the degree of active anterior elevation, according to the UCLA scale and to patient satisfaction. Results: Pre-operative 
active anterior elevation had a mean of $102^{\circ}$, and the degree of active anterior elevation after surgery presented a mean of $175^{\circ}$. The mean gain in active anterior elevation was $73^{\circ}(p<0.0001)$. Preoperative UCLA had a mean of 15.25 score points, and after surgery, a mean of 31.66 score points. The mean UCLA index was 16 score points ( $p<0.0001) ; 10$ excellent results, one good, and one poor. Satisfactory results: 10, unsatisfactory: 1. Conclusion: 1) The arthroscopic suture of the subscapularis lesions presents satisfactory results in $91.67 \%$ of the cases for isolated upper third lesions and for total complete lesions; 2) biceps instability is a common finding; 3) arthroscopic suture of the subscapularis is possible even in retracted tendons; 4) the technique is safe; 5) complete lesions of the upper third of the subscapularis are symptomatic and should be treated as complete lesions.

\section{Keywords - Shoulder/injuries; Rotator cuff/injuries; Arthroscopy; Retrospective studies}

\section{INTRODUÇÃO}

As lesões do subescapular costumam ser encontradas em associação com a ruptura de outros tendões do manguito rotador, sendo sua lesão isolada menos freqüente e, geralmente, de origem traumática ${ }^{(1)}$. Existem alguns relatos sobre os resultados do reparo aberto destas lesões ${ }^{(2-4)}$. Entretanto, estudos atuais vêm mostrando a possibilidade da sutura artroscópica com resultados promissores ${ }^{(5-6)}$. Segundo Bennett, o tratamento artroscópico destas lesões gera expectativas seguras quanto à melhora da dor e restauração da função da articulação do ombro ${ }^{(7)}$.

As lesões do tendão do subescapular podem ser classificadas segundo a profundidade, a extensão e o grau de retração dos tendões. Pela profundidade, podem ser parciais, com comprometimento apenas das fibras articulares, ou totais, atingindo toda a superfície do tendão. A inserção do músculo subescapular possui uma porção tendinosa, correspondente a seu terço superior, e uma porção musculoligamentar-capsular, que são os dois terços inferiores do tendão. De acordo com a extensão, as lesões podem comprometer o terço superior ou toda a extensão do tendão. Por sua vez, as rupturas completas podem ser sem retração, geralmente com o ligamento coracoumeral com seus feixes lateral e medial intactos, ou com tendão retraído, associados à lesão dos dois feixes do ligamento coracoumeral ${ }^{(8)}$.

Neste estudo, temos como objetivo avaliar os resultados retrospectivos de 12 pacientes submetidos ao reparo artroscópico de lesões completas isoladas do subescapular após seguimento mínimo de um ano.

\section{MÉTODOS}

De 11 de abril de 2002 a 24 de setembro de 2004, realizamos o reparo artroscópico de lesões completas do manguito rotador em 95 pacientes (96 ombros) no Instituto de Ortopedia Niso Balsini - Joinville/SC. Destes, 31 (32,29\%) pacientes apresentam comprometimento do subescapular, sendo $12(12,5 \%)$ lesões isoladas compondo o grupo de estudo. Estes foram reavaliados após seguimento mínimo de um ano da cirurgia, de acordo com o grau de elevação anterior ativa, escala da UCLA, e lhes foi perguntado se aceitariam novo tratamento (tabela 1 ).

Dos 12 pacientes submetidos à cirurgia artroscópica, sete eram do sexo masculino e cinco do feminino; a idade variou de 41 a 71 anos, com média de 52,4 anos. Quanto ao lado dominante, 11 eram destros e um canhoto, sendo que, dos 12 pacientes, 10 foram operados no lado direito e dois no esquerdo. Dos tipos de lesões encontradas, sete eram de origem traumática e cinco, de origem degenerativa; em seis casos a lesão encontrava-se no terço superior do tendão e seis apresentavam lesão completa total. Duas das lesões completas totais eram retraídas.

Para a fixação do tendão do subescapular, foram utilizadas de uma a três âncoras com média de 2,08. Em sete casos realizamos a tenotomia da cabeça longa do músculo bíceps braquial (CLMBB); em quatro, a tenotomia com tenodese; e, em um caso, não houve qualquer procedimento neste tendão. Quanto aos procedimentos de descompressão, efetuou-se acromioplastia em cinco casos e coracoplastia em quatro.

O grau de elevação anterior ativa pré-operatória (EAA pré) variou de $20^{\circ}$ a $175^{\circ}$, com média de $102,1^{\circ}$, e o 


\section{QUADRO 1}

Dados dos pacientes

\begin{tabular}{|cccccccc|}
\hline Pacientes & $\begin{array}{c}\text { Idade } \\
\text { anos }\end{array}$ & Sexo & $\begin{array}{c}\text { Lado } \\
\text { dominante }\end{array}$ & $\begin{array}{c}\text { Lado } \\
\text { operado }\end{array}$ & $\begin{array}{c}\text { Tipo de } \\
\text { lesão }\end{array}$ & $\begin{array}{c}\text { Classificação } \\
\text { da lesão }\end{array}$ & $\begin{array}{c}\text { Número de } \\
\text { âncoras }\end{array}$ \\
\hline 1 & 48 & F & E & D & Trauma & Total & 2 \\
2 & 49 & M & D & D & Trauma & Total & 2 \\
3 & 52 & F & D & D & Degenerativa & Total & 1 \\
4 & 44 & F & D & D & Trauma & Total & 2 \\
5 & 53 & M & D & D & Trauma & $1 / 3$ superior & 2 \\
6 & 54 & M & D & E & Degenerativa & $1 / 3$ superior & 2 \\
7 & 47 & F & D & D & Degenerativa & $1 / 3$ superior & 2 \\
8 & 45 & M & D & D & Trauma & $1 / 3$ superior & 2 \\
9 & 71 & F & D & D & Degenerativa & $1 / 3$ superior & 2 \\
10 & 63 & M & D & D & Degenerativa & $1 / 3$ superior & 2 \\
11 & 62 & M & D & E & Trauma & Total & 3 \\
12 & 41 & M & D & D & Trauma & Total & 3 \\
\hline
\end{tabular}

M: Masculino; F: Feminino; E: Esquerdo; D: Direito; Trauma: Traumática. Fonte: Instituto Balsini - 2008

TABELA 1

Procedimentos e avaliação clínica

\begin{tabular}{ccccc}
\hline Bíceps & Acromioplastia & Coracoplastia & $\begin{array}{c}\text { Elevação anterior } \\
\text { pré-operatória } \\
\text { (graus) }\end{array}$ & $\begin{array}{c}\text { UCLA } \\
\text { pré-operatória } \\
\text { (pontos) }\end{array}$ \\
\hline Tenotomia & Sim & Não & $90^{\circ}$ & 11 \\
Tenotomia & Sim & Não & $60^{\circ}$ & 10 \\
Tenotomia & Sim & Não & $150^{\circ}$ & 20 \\
Tenotomia & Não & Não & $20^{\circ}$ & 17 \\
Tenotomia & Não & Não & $60^{\circ}$ & 16 \\
Tenotomia + tenodese & Não & Não & $170^{\circ}$ & 21 \\
Tenotomia + tenodese & Sim & Não & $100^{\circ}$ & 14 \\
Tenotomia & Não & Sim & $90^{\circ}$ & 17 \\
Tenotomia + tenodese & Sim & Sim & $100^{\circ}$ & 12 \\
Tenotomia + tenodese & Não & Sim & $175^{\circ}$ & 22 \\
Tenotomia & Não & Sim & $120^{\circ}$ & 10 \\
\hline
\end{tabular}

Fonte: Instituto Balsini - 2008

índice da UCLA pré-operatório, de 10 a 22 pontos, com média de 15,25. O grupo de pacientes com lesão do terço superior do subescapular (seis casos) apresentou grau EAA pré média de $115^{\circ}\left(60^{\circ}-175^{\circ}\right)$ e índice da UCLA com 17 pontos (16-22); já o grau de EAA pré e o índice da UCLA do grupo com lesão completa (seis pacientes) foram de $88^{\circ}\left(20^{\circ}-150^{\circ}\right)$ e 13,5 pontos $(10$ 20), respectivamente (tabela 2 ).

O método ANOVA de fator único foi empregado para análise estatística dos resultados.

\section{Técnica cirúrgica}

Os pacientes foram submetidos a anestesia geral associada a bloqueio de plexo braquial ou infiltração articular $(10 \mathrm{ml})$ e subacromial $(10 \mathrm{ml})$ de uma solução contendo $10 \mathrm{ml}$ de cloridrato de bupivacaína a $0,50 \%$ com vasoconstritor e $10 \mathrm{ml}$ de soro fisiológico. Cada paciente foi posicionado em decúbito lateral, com o lado operado em tração longitudinal e transversal.

A artroscopia foi iniciada pelo portal posterior, seguindo-se a avaliação articular, identificação das le- 
sões associadas e classificação da lesão do tendão do subescapular propriamente dito (tabela 2 e figura 1 ).

Realizamos um portal ântero-superior para avaliação, reparo e tenotomia do tendão do bíceps (figura 2).

Avaliamos com uma lâmina de shaver de 5,5mm o tamanho do espaço subcoracóide e, quando observa-

TABELA 2

Classificação das lesões do tendão do subescapular

\section{Profundidade das lesões}

A. Totais: ruptura de toda a profundidade

B. Parciais: ruptura parcial da profundidade

\section{Extensão das lesões}

A. Completa: de toda a extensão

B. Parciais: do terço superior

\section{Retração das lesões}

A. Com retração: associadas a lesões dos ligamentos coracoumeral medial e lateral

B. Sem retração: ligamentos coracoumerais intactos

Fonte: Traduzido de: Bennett WF. Arthroscopic repair of isolated subscapularis tears: A prospective cohort with 2 - to 4 - year follow-up. Arthroscopy. 2003:19(2):131-43(18).
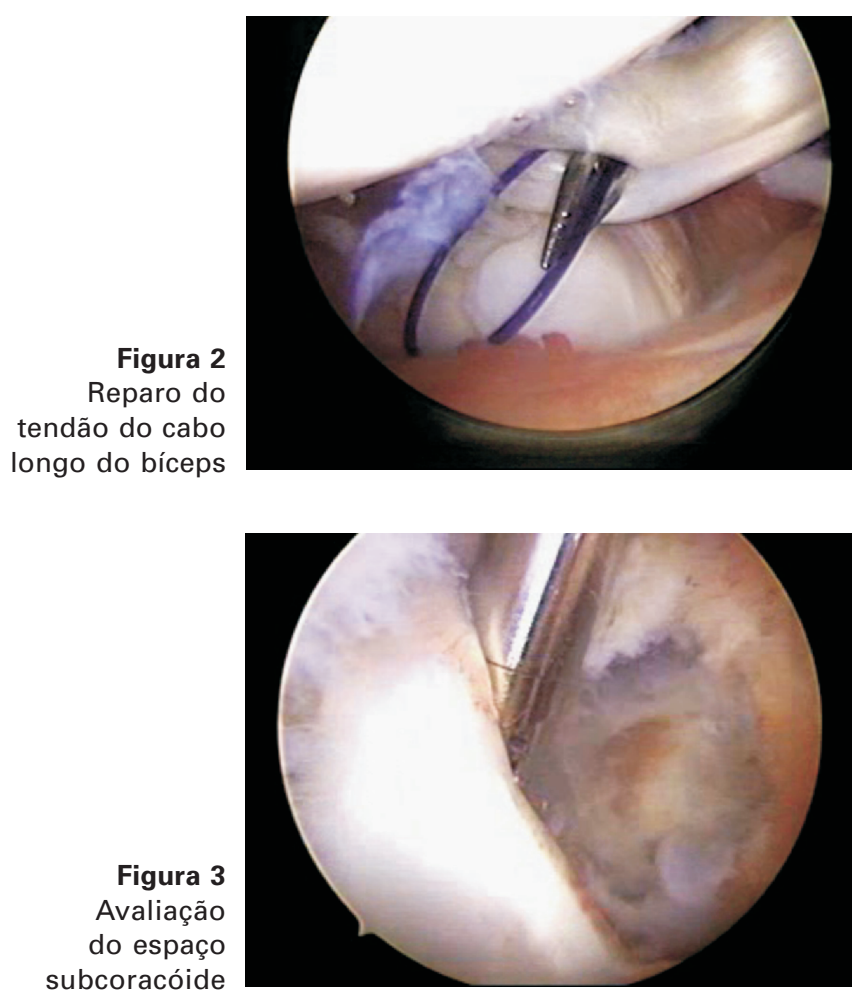

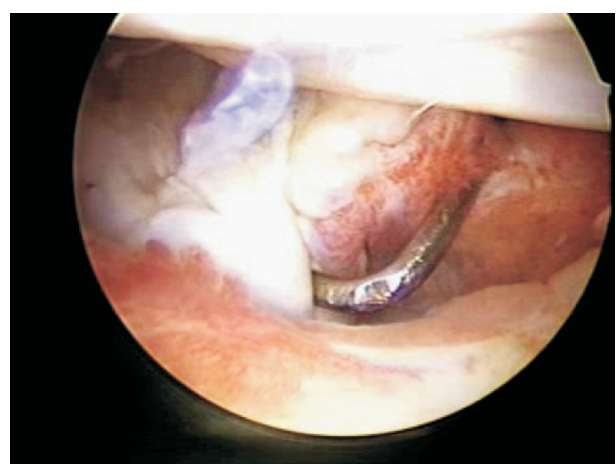

Figura 1A Identificação da lesão do subescapular visão articular frontal.

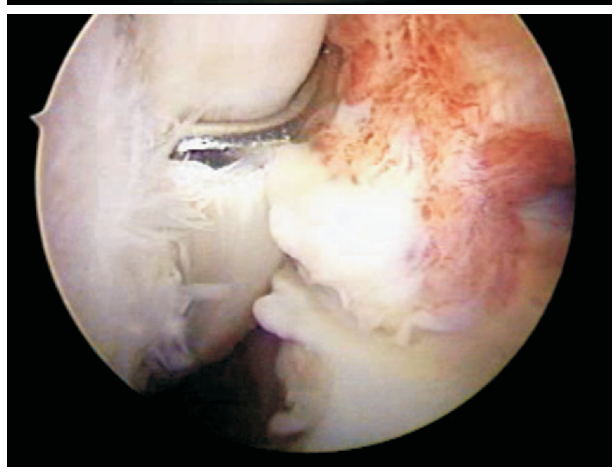

Figura 1B

Ausência de inserção do tendão do subescapular - footprint confirmando a lesão completa.

mos tamanho menor que 6,0mm, aproximadamente, realizamos a coracoplastia (figura 3 ).

O preparo do local da reinserção do tendão e a introdução das âncoras foram realizados pelo portal ântero-superior (figura 4). Na sutura do tendão, quando realizada de maneira retrógrada, utilizamos o portal ântero-inferior; já na sutura com pinça "Caspari”, passamos o ponto utilizando o portal lateral acessório. Os nós foram dados pelo portal ântero-superior e lateral acessório (figuras 5 e 6).

Posteriormente à fixação do subescapular, realizamos a bursoscopia. Nesta etapa realizamos a tenodese

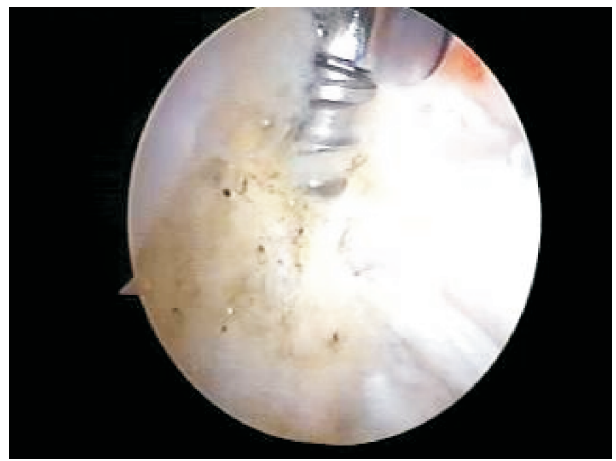

Figura 4A Introdução da âncora através do portal ânterosuperior 


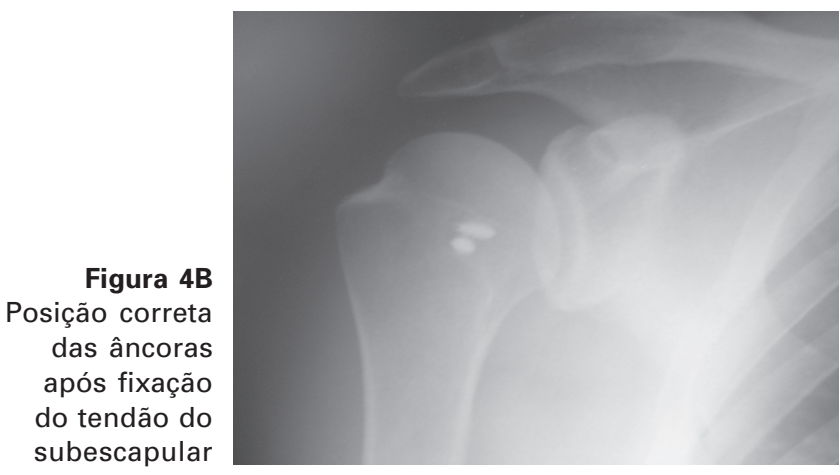

da CLMBB (nos casos de tenotomia e tenodese - quatro pacientes) com âncoras e a acromioplastia, quando na presença de sinais de impacto subacromial associado a esporão ântero-inferior do acrômio (cinco pacientes).

\section{RESULTADOS}

Os pacientes foram reavaliados após período de 12 a 35 meses, com média de 17,25 meses.

O grau de elevação anterior ativa pré-operatória foi de $20^{\circ}$ a $175^{\circ}$, com média de $102^{\circ}$, enquanto o grau de elevação anterior pós-operatória (EAA pós) foi de $150^{\circ}$ a $180^{\circ}$, com média de $175^{\circ}$. Observamos ganho na elevação anterior ativa média de $73^{\circ}(\mathrm{p}<0,0001)$ (tabela 3).

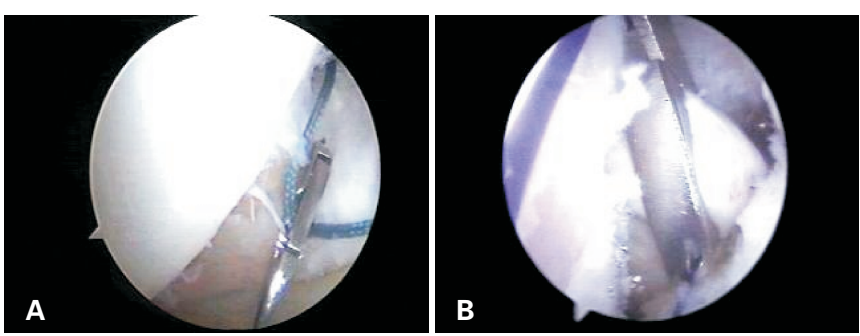

Figura 5 - A) Sutura retrógrada do subescapular. B) Sutura do subescapular com pinça "Caspari" - sutura anterógrada.
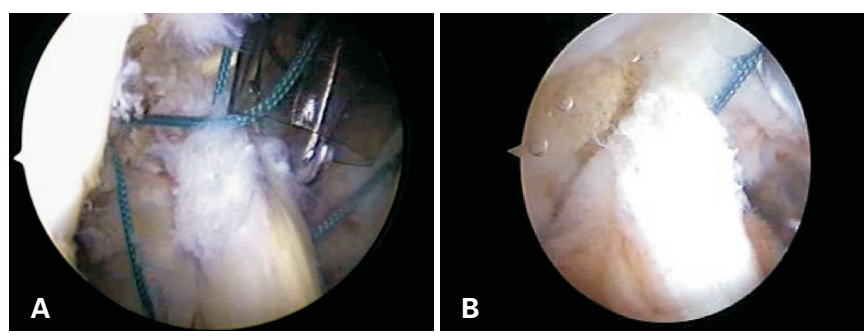

Figura 6 - A) Os nós são realizados através do portal ântero-superior. B) Fixação final do tendão.

A UCLA pré-operatória foi de 10 a 20 pontos, com média de 15,25 pontos e, no pós-operatório, foi observada variação de 15 a 35 pontos, com média de 31,66 pontos. O ganho médio do índice da UCLA entre pré e pós-operatório foi de 16 pontos ( $\mathrm{p}<0,0001)$, com 10 resultados excelentes, um bom e um mau. Dos resultados, 11 foram considerados satisfatórios $(91,67 \%)$ e um insatisfatório $(8,33 \%)$ (tabela 3$)$.

TABELA 3

Dados clínicos comparativos

\begin{tabular}{rcccccc}
\hline Paciente & $\begin{array}{c}\text { Seguimento } \\
\text { (meses) }\end{array}$ & $\begin{array}{c}\text { Elevação } \\
\text { anterior ativa } \\
\text { pré-operatória } \\
\text { (graus) }\end{array}$ & $\begin{array}{c}\text { Elevação } \\
\text { anterior ativa } \\
\text { pós-operatória } \\
\text { (graus) }\end{array}$ & $\begin{array}{c}\text { UCLA } \\
\text { pré-operatório } \\
\text { (pontos) }\end{array}$ & $\begin{array}{c}\text { UCLA } \\
\text { pós-operatório } \\
\text { (pontos) }\end{array}$ & Questionário \\
\hline 1 & 35 & $90^{\circ}$ & $180^{\circ}$ & 11 & 35 & Sim \\
2 & 30 & $60^{\circ}$ & $175^{\circ}$ & 10 & 33 & Sim \\
3 & 14 & $150^{\circ}$ & $175^{\circ}$ & 20 & 33 & Sim \\
4 & 18 & $20^{\circ}$ & $180^{\circ}$ & 17 & 33 & Sim \\
5 & 15 & $60^{\circ}$ & $180^{\circ}$ & 16 & 33 & Sim \\
6 & 14 & $170^{\circ}$ & $160^{\circ}$ & 21 & 15 & Não \\
7 & 18 & $100^{\circ}$ & $180^{\circ}$ & 14 & 35 & Sim \\
8 & 15 & $90^{\circ}$ & $180^{\circ}$ & 17 & 33 & Sim \\
9 & 12 & $100^{\circ}$ & $180^{\circ}$ & 12 & 33 & Sim \\
10 & 12 & $175^{\circ}$ & $180^{\circ}$ & 22 & 35 & Sim \\
11 & 12 & $120^{\circ}$ & $150^{\circ}$ & 10 & 28 & Sim \\
12 & 12 & $90^{\circ}$ & $180^{\circ}$ & 13 & 34 & Sim \\
\hline
\end{tabular}

Fonte: Instituto Balsini - 2008 
Com relação ao questionário aplicado a cada paciente após a cirurgia, dos 12 pacientes que fizeram parte do grupo de estudo, um estava insatisfeito e não faria novamente a cirurgia. Este paciente apresentou índice da UCLA de 15 e persiste afastado do trabalho com mais de dois anos de pós-operatório e com problemas litigiosos.

O grupo de pacientes com lesão do terço superior do subescapular (seis casos) apresentou grau de elevação anterior média pós-operatória de $176^{\circ}\left(160^{\circ}-180^{\circ}\right)$ e o grupo com lesão completa total de $173^{\circ}\left(150^{\circ}-180^{\circ}\right)$. Não foi demonstrada diferença estatística significativa entre os grupos com lesão do terço superior e lesão completa $(\mathrm{p}=0,57)$. O índice da UCLA pós-operatório dos pacientes com lesão do terço superior foi de 31 pontos (15-35) e do grupo com lesão completa total de 33 pontos (28-35), também sem diferença estatística significativa $(\mathrm{p}=0,55)$ (tabela 4$)$.

Como complicação, em um dos pacientes com tenotomia simples da CLMBB ocorreu a soltura do tendão do bíceps, evoluindo com abaulamento na face anterior do braço.

\section{DISCUSSÃO}

As lesões do tendão do subescapular são freqüentes e devem ser lembradas durante a avaliação do ombro doloroso. O comprometimento do pilar anterior após lesão completa do subescapular tem como consequiência perda funcional importante, estando o tratamento

TABELA 4

Resultado comparativo das lesões parciais e completas

\begin{tabular}{cccc}
\hline $\begin{array}{c}\text { Elevação } \\
\text { anterior } \\
\text { ativa das } \\
\text { lesões do } \\
\begin{array}{c}\text { terço superior } \\
\text { (graus) }\end{array}\end{array}$ & $\begin{array}{c}\text { Elevação } \\
\text { anterior } \\
\text { ativa das } \\
\text { lesões } \\
\text { completas } \\
\text { (graus) }\end{array}$ & $\begin{array}{c}\text { UCLA das } \\
\text { lesões } \\
\text { do terço } \\
\text { superior } \\
\text { (graus) }\end{array}$ & $\begin{array}{c}\text { UCLA das } \\
\text { lesões } \\
\text { completas } \\
\text { (graus) }\end{array}$ \\
\hline $180^{\circ}$ & $180^{\circ}$ & $33^{\circ}$ & $35^{\circ}$ \\
$160^{\circ}$ & $175^{\circ}$ & $15^{\circ}$ & $33^{\circ}$ \\
$180^{\circ}$ & $175^{\circ}$ & $35^{\circ}$ & $33^{\circ}$ \\
$180^{\circ}$ & $180^{\circ}$ & $33^{\circ}$ & $33^{\circ}$ \\
$180^{\circ}$ & $150^{\circ}$ & $33^{\circ}$ & $28^{\circ}$ \\
$180^{\circ}$ & $180^{\circ}$ & $35^{\circ}$ & $34^{\circ}$ \\
\hline $\mathrm{p}=0,57$ & & $\mathrm{p}=0,55$ & \\
\hline
\end{tabular}

Fonte: Instituto Balsini - 2008

TABELA 5

Avaliação da amplitude de movimento pré e pós-operatória

\begin{tabular}{crrrrrr}
\hline Paciente & EAA pré & EAA pós & RE pré & RE pós & RI pré & RI pós \\
\hline 1 & $90^{\circ}$ & $180^{\circ}$ & $70^{\circ}$ & $60^{\circ}$ & $\mathrm{T} 8$ & $\mathrm{~T} 7$ \\
2 & $60^{\circ}$ & $175^{\circ}$ & $80^{\circ}$ & $60^{\circ}$ & $\mathrm{T} 4$ & $\mathrm{~T} 4$ \\
3 & $150^{\circ}$ & $175^{\circ}$ & $80^{\circ}$ & $80^{\circ}$ & $\mathrm{T} 5$ & $\mathrm{~T} 2$ \\
4 & $20^{\circ}$ & $180^{\circ}$ & $90^{\circ}$ & $80^{\circ}$ & $\mathrm{T} 5$ & $\mathrm{~T} 4$ \\
5 & $60^{\circ}$ & $180^{\circ}$ & $60^{\circ}$ & $60^{\circ}$ & $\mathrm{T} 5$ & $\mathrm{~T} 4$ \\
6 & $170^{\circ}$ & $160^{\circ}$ & $70^{\circ}$ & $60^{\circ}$ & $\mathrm{T} 7$ & $\mathrm{~T} 4$ \\
7 & $100^{\circ}$ & $180^{\circ}$ & $70^{\circ}$ & $70^{\circ}$ & $\mathrm{T} 5$ & $\mathrm{~T} 5$ \\
8 & $90^{\circ}$ & $180^{\circ}$ & $40^{\circ}$ & $60^{\circ}$ & $\mathrm{T} 5$ & $\mathrm{~T} 5$ \\
9 & $100^{\circ}$ & $180^{\circ}$ & $50^{\circ}$ & $60^{\circ}$ & $\mathrm{T} 5$ & $\mathrm{~T} 4$ \\
10 & $175^{\circ}$ & $180^{\circ}$ & $60^{\circ}$ & $60^{\circ}$ & $\mathrm{T} 4$ & $\mathrm{~T} 4$ \\
11 & $120^{\circ}$ & $150^{\circ}$ & $55^{\circ}$ & $50^{\circ}$ & $\mathrm{T} 6$ & $\mathrm{~T} 5$ \\
12 & $90^{\circ}$ & $180^{\circ}$ & $80^{\circ}$ & $80^{\circ}$ & $\mathrm{T} 6$ & $\mathrm{~T} 6$ \\
Média & $102^{\circ}$ & $175^{\circ}$ & $67^{\circ}$ & $65^{\circ}$ & 5,42 & 4,5
\end{tabular}

Fonte: Instituto Balsini - 2008

Legenda:

EAA pré - elevação anterior ativa pré-operatória (em graus)

EAA pós - elevação anterior ativa pós-operatória (em graus)

RE pré - rotação externa pré-operatória (vértebra alcançada)

RE pós - rotação externa pós-operatória (vértebra alcançada)

RI pré - rotação interna pré-operatória

RI pós - rotação interna pós-operatória 
cirúrgico indicado. Não temos número significativo de artigos no que se refere aos resultados do tratamento cirúrgico nas lesões isoladas, mas na maioria deles são satisfatórios ${ }^{(2-4)}$. Bennett publicou os resultados da sutura artroscópica de lesões isoladas do subescapular em oito pacientes com seguimento de dois anos; todos eles apresentaram melhora dos índices de avaliação do ombro e se encontravam satisfeitos com a cirurgia ${ }^{(7)}$. Em nossa casuística, apenas um paciente não estava satisfeito e encontrava-se pior com o tratamento. $\mathrm{O}$ mesmo estava envolvido em problemas litigiosos.

Podemos observar que os pacientes com lesões do terço superior do tendão subescapular geralmente não cursam com os sinais e sintomas clássicos de insuficiência deste tendão, mas apresentam queixa de dor na região anterior do ombro e testes para avaliação da CLMBB, positivos. O "teste de Napoleão" é maneira bastante sensível para pesquisar este tipo de lesão( ${ }^{(5)}$. Classicamente, pode ser considerado negativo, intermediário e positivo. Geralmente, as lesões do terço superior classificam-se como intermediárias, ou seja, ocorre a flexão do punho de 30 a $60^{\circ}$ durante a compressão abdominal.

O espaço entre o processo coracóide e o tubérculo menor é denominado intervalo coracoumeral. A estenose deste espaço tem sido interpretada como uma das causas de dor anterior do ombro e como etiologia das lesões do subescapular ${ }^{(9-10)}$. Estudos de imagem e anatômicos mostram que, normalmente, este espaço é de 8,4 a $11 \mathrm{~mm}^{(11-13)}$. Lo et al definiram como estenose subcoracóide a condição em que este espaço encontra-se menor do que seis milímetros e sugerem o impacto subcoracóide como uma das etiologias das lesões do subescapular em conseqüência do mecanismo denominado de "efeito rolo compressor"(10). Em nosso estudo, encontramos a diminuição deste espaço em quatro casos nos quais realizamos a coracoplastia através do intervalo rotador. A descompressão deste espaço facilita também a abordagem intra-operatória das lesões do subescapular; assim, na presença de estenose, consideramos importante a coracoplastia antes de iniciar a sutura do tendão.

A maioria das lesões com comprometimento do subescapular apresenta também comprometimento de outros tendões do manguito rotador, sendo a lesão isolada menos freqüente. Em nossa casuística, 31 lesões com comprometimento do subescapular, apenas 12 eram isoladas, sendo seis do terço superior e seis completas. No estudo de Bennett, de 47 lesões do subescapular, oito eram lesões isoladas, correspondendo a seis lesões completas e duas do terço superior ${ }^{(7)}$.

Quando comparamos os resultados da sutura artroscópica do grupo com lesão do terço superior com o grupo com lesão completa, não observamos diferença estatística no que se refere ao grau de elevação anterior ativa e índice da UCLA pós-operatório. Consideramos importantes a pesquisa e a avaliação do subescapular para identificar lesões do terço superior e estas merecem ser abordadas da mesma maneira que as lesões completas.

A tendinite, a subluxação e a luxação da CLMBB comumente estão associadas às lesões do tendão do subescapular, podendo ser tratadas com tenotomia, desbridamento ou tenodese. Bennett defendeu a reconstrução do complexo ligamentar medial da CLMBB associada à sutura do subescapular sem tenotomia ${ }^{(7-8)}$. Burkhart et al preferem realizar sempre a tenotomia e a tenodese, pois acreditam que a possibilidade de soltura da fixação da polia bicipital pode comprometer a fixação do subescapular(5). Dos 12 pacientes deste estudo, 11 apresentavam lesão da polia bicipital com instabilidade da CLMBB, necessitando tenotomia. Destes, em sete casos realizamos a tenotomia pura e simples e, em quatro, a tenodese, sendo que, nos primeiros pacientes da série, utilizamos de rotina a tenotomia sem fixação e, no decorrer do estudo, passamos a realizar a tenodese com âncoras sempre quando tenotomizamos o bíceps, independente do grau de degeneração do mesmo ou da idade do paciente. Em um dos pacientes, não encontramos lesão da polia bicipital (instabilidade). Um dos pacientes submetidos à tenotomia sem tenodese da CLMBB evoluiu com migração distal do coto proximal necessitando de um segundo procedimento para tenodese da CLMBB através de miniincisão subpeitoral. Este paciente apresentou resultado excelente durante a evolução; esta complicação não comprometeu o reparo do subescapular. 
Devido à proximidade de estruturas neurovasculares ao tendão do subescapular, alguns autores defendem a cirurgia aberta pelo acesso anterior nas lesões retraídas $^{(6)}$. Checchia et al apresentaram resultados satisfatórios utilizando a abordagem deltopeitoral no tratamento das lesões ântero-superiores (supra-espinal e subescapular), mostrando ser um acesso seguro $^{(14)}$. Outros autores defenderam a segurança da artroscopia, determinando alguns limites anatômicos para trabalhar nas proximidades do processo coracói$\mathrm{de}^{(15-16)}$. Neste estudo, dois pacientes apresentavam retração importante do coto tendinoso do subescapular, necessitando liberação e mobilização do tendão para fixação; nenhum dos casos apresentou complicações.

Anteriormente utilizamos a técnica de sutura artroscópica das lesões do subescapular trabalhando pelos espaços articular e bursal ${ }^{(17)}$. Nas lesões isoladas do subescapular, a visualização do tendão pelo espaço bursal é prejudicada devido à integridade do tendão do supra-espinal; assim, preferimos fazer a sutura destas lesões trabalhando pelo lado articular.

\section{CONCLUSÕES}

A sutura artroscópica das lesões do subescapular apresentou resultados satisfatórios em 91,67\% dos casos, tanto para as lesões isoladas do terço superior quanto para as lesões completas. A instabilidade do bíceps foi comumente encontrada devido à lesão da polia medial secundaria à rotura do subescapular. A sutura artroscópica do subescapular foi possível mesmo nas lesões retraídas; demonstrou ser técnica segura, com número pequeno de complicações. As lesões completas do terço superior do subescapular são sintomáticas e devem ser tratadas como tal.

\section{REFERÊNCIAS}

1. Lo IK, Parten PM, Burkhart SS. Combined subcoracoid and subacromial impingement in association with anterosuperior rotator cuff tears: An arthroscopic approach. Arthroscopy. 2003;19(10):1068-78.

2. Gerber C, Krushnell RJ. Isolated rupture of the tendon of the subscapularis muscle: Clinical features in 16 cases. J Bone Joint Surg Br. 1991;73(3):389-94.
3. Gerber C, Hersche O, Farron A. Isolated rupture of the subscapularis tendon. J Bone Joint Surg Am. 1996;78(7):101523.

4. Ticker JB, Warner JJ. Single-tendon tears of the rotator cuff. Evaluation and treatment of subscapularis tears and principles of treatment for supraspinatus tears. Orthop Clin North Am. 1997;28(10):99-116.

5. Burkhart SS, Tehrany AM. Arthroscopic subscapularis tendon repair: technique and preliminary results. Arthroscopy. 2002; 18(5):454-63.

6. Esch JC, Bynum CK. Arthroscopic repair of subscapularis tendon ruptures. Sports Med Arthrosc. 2004;12(1):99-103.

7. Bennett WF. Arthroscopic repair of massive rotator cuff tears: a prospective cohort with 2- to 4-year follow-up. Arthroscopy. 2003;19(4):380-90.

8. Bennett WF. Subscapularis, medial, and lateral head coracohumeral ligament insertion anatomy: Arthroscopic appearance and incidence of "hidden" rotator interval lesions. Arthroscopy. 2001;17(2):173-80.

9. Simoni M, Lech O. O tratamento cirúrgico da síndrome impacto idiopática no processo coracóide. Rev Bras Ortop. 2004;39(1/ 2):14-20.

10. Lo IK, Burkhart SS. The etiology and assessment of subscapularis tendon tears: a case for subcoracoid impingement, the roller-wringer effect, and the TUFF lesions of the subscapularis. Arthroscopy. 2003;19(10):1142-50.

11. Gerber C, Terrier F, Zehnder R, Ganz R. The subcoracoid space: An anatomic study. Clin Orthop Relat Res. 1987;(215): 132-8.

12. Friedman RJ, Bonutti PM, Genez B. Cine magnetic resonance imaging of the subcoracoid region. Orthopedics. 1998;21(5): 545-8.

13. Nové-Josserand L, Boulahia A, Levigne C, Noel E, Walch G. Coraco-humeral space and rotator cuff tears. Rev Chir Orthop Reparatrice Appar Mot. 1999;85(7):677-83.

14. Checchia SL, Doneux PS, Miyazaki AN, Fregoneze M, Silva LA, Mussi Filho S, Faria AC, Ribeiro Jr GFF. Tratamento cirúrgico das lesões extensas do manguito rotador pela via de acesso deltopeitoral. Rev Bras Ortop. 2003;38(5):252-60.

15. Lo IK, Burkhart SS, Parten PM. Surgery about the coracoid: neurovascular structures at risk. Arthroscopy. 2004;20(6):5915.

16. Wright JM, Heavrin B, Hawkins RJ, Noonan T. Arthroscopic visualization of the subscapularis tendon. Arthroscopy. 2001; 17(7):677-84.

17. Balsini NE, Balsini N, Jacintho PM, Kochen L. Reparo artroscópico das lesões completas do tendão do músculo subescapular: abordagem combinada articular e bursal, apresentação da técnica e resultados preliminares. Rev Bras Ortop. 2005;40(10): 582-89.

18. Bennett WF. Arthroscopic repair of isolated subscapularis tears: A prospective cohort with 2 - to 4 - year follow-up. Arthroscopy. 2003;19(2):131-43. 